\title{
O potencial transformador do comum em saúde
}

\author{
The transformative potential of health as a commons
}

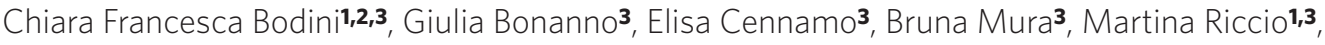 \\ Lorenza Santoro 3
}

DOI: 10.1590/0103-11042020S107

RESUMO A Itália foi um dos países participantes do projeto de pesquisa-ação multicêntrica do Movimento pela Saúde dos Povos (Peoples's Health Movement), chamado 'Engajamento da Sociedade Civil para a Saúde para Todos' (Civil Society Engagement for Health for All). A equipe italiana, um coletivo chamado Grup-pa, realizou várias atividades participativas de pesquisa-ação, incluindo, em uma primeira fase, um mapeamento de grupos ativos em áreas ligadas à determinação social da saúde e à promoção da saúde, através de entrevistas individuais e coletivas. Em uma segunda fase, três oficinas públicas, estruturadas em torno do intercâmbio de práticas, focalizaram-se em temas-chave surgidos durante a primeira fase. Um importante construto originado deste trabalho, centrado em torno da co-construção do conhecimento experiencial do comum em saúde, foi denominado 'práticas do comum em saúde'. O foco nas práticas não é meramente estratégico (produzir sinergias e alianças), mas inerentemente político (conceber a participação como um valor) e ligado à saúde e à manutenção da saúde (dos indivíduos; da comunidade). O conceito de 'práticas do comum em saúde' pretende tornar visível uma área de transformações contínuas em novos espaços criados pelos movimentos sociais e em ações mais tradicionais em defesa dos serviços públicos existentes, abordando a saúde como uma questão sociopolítica. Neste ensaio, esboça-se uma reflexão em torno de seis palavras-chave que lhe são centrais: comum, cuidado, tecnologia, eficácia, sustentabilidade, instituição.

PALAVRAS-CHAVE Promoção da saúde. Mudança social. Equidade. Participação social.

1 University of Bologna, Centre for International and Intercultural Health (CSI) - Bologna, Italy. chiara.bodini@unibo.it

2 Viva Salud - Brussels, Belgium.

3 People's Health Movement (PHM) -

Bologna, Italy.
ABSTRACT Italy was a participating country in the People's Health Movement multi-centred actionresearch project (Civil Society Engagement for Health for All). The Italian team, a collective named Grup-pa, undertook several participatory action-research activities including, in a first phase, a mapping of groups active in fields linked to the social determination of health and health promotion, through individual and collective interviews. In a second phase, three public workshops, structured around the exchange of practices, focused on key themes emerged from phase one. A major construct originated from this work, centred around the co-construction of experiential knowledge on health as a commons, has been named 'health commons practices'. The focus on practices is not merely strategic (producing synergies and alliances), but inherently political (conceiving participation as a value) and connected to health and staying healthy (as individuals; as a community).The construct of 'health commons practices' is meant to make visible an area of ongoing transformations in new spaces created by movements and in more traditional actions in defence of existing public services, addressing health as a socio-political issue. In this essay, we sketch the reflection around six keywords that are central to it: commons, care, technology, efficacy, sustainability, institution.

KEYWORDS Health promotion. Social change. Equity. Social participation. 


\section{Introdução}

Entre os anos de 2014 e 2018, o Movimento pela Saúde dos Povos (People's Health Movement - 'PHM') realizou um grande projeto de pesquisa-ação participativa e multicêntrica explorando o ativismo de movimentos sociais na luta pela 'saúde para todos', incluindo ações sobre os determinantes sociais da saúde e acesso a sistemas de saúde acessíveis e de alta qualidade. $\mathrm{O}$ objetivo da pesquisa era entender melhor o engajamento da sociedade civil em cinco domínios da saúde (criação de movimentos; campanha e ativismo; desenvolvimento de capacidades; geração, uso e acesso ao conhecimento; e interação com o governo), com o objetivo de melhorar a prática ativista.

A Itália foi um dos seis países que participaram do projeto, que foi realizado por um coletivo chamado Grup-pa (acrônimo de 'grupo permanentemente aberto'). O coletivo foi criado especificamente para o projeto e foi formado por uma rede de pessoas - em sua maioria, oriundas da área da saúde - que tinham colaborado anteriormente para a defesa de mudanças na educação médica, com maior enfoque na determinação social da saúde e na relação entre os processos de globalização e a desigualdade na saúde ${ }^{\mathbf{1 , 2}}$. A rede também tinha promovido atividades de formação auto-organizadas em diversas universidades italianas e em nível nacional, e contribuído para a criação da Rede Italiana para o Ensinamento da Saúde Global (RIISG) ${ }^{3}$.

O objetivo principal do Grup-pa ao aderir a um projeto de pesquisa-ação mais vasto era contribuir para a criação e o fortalecimento de um movimento pela saúde na Itália. Isso foi baseado em dois pressupostos: 1) A crença - fundamentada na história e na experiência - de que mudanças ocorrem quando as pessoas mais preocupadas com uma questão se organizam e assumem um papel ativo na tentativa de enfrentá-la ${ }^{4-6} ; 2$ ) A alta fragmentação entre os movimentos sociais na Itália e a ausência de um movimento pela saúde (apesar de uma rica história que levou à criação de um sistema universal de saúde, em 1978, juntamente à aprovação de leis progressistas sobre saúde reprodutiva e mental). A metodologia da pesquisa-ação participativa foi, neste sentido, muito bem colocada para atuar no âmbito da geração de conhecimento e de ação e prática, já que uma condição prévia para o envolvimento do coletivo era a intenção de promover uma mudança rumo a uma sociedade mais justa, igualitária e saudável7.

Juntamente à estrutura principal do projeto, a pesquisa-ação italiana desenvolveu-se em duas fases. Em uma primeira fase, o Grup-pa realizou um mapeamento dos grupos ativos na Itália em campos ligados à determinação social da saúde e à promoção da saúde, através de entrevistas individuais e coletivas. Em uma segunda fase, o coletivo organizou e facilitou três oficinas públicas estruturadas em torno do intercâmbio de práticas através de metodologias inclusivas e participativas, focalizadas em eixos temáticos-chave surgidos a partir da análise dos dados coletados na primeira fase: movimentos sociais e bem-estar social; construção de espaços e comunidades saudáveis através da reapropriacão e da auto-organização coletivas; novas formas de comunidade e sustentabilidade através do comum.

O resultado principal deste trabalho, centrado na co-construção do conhecimento experiencial em saúde comunitária, recebe o nome de 'práticas do comum em saúde'. Esse conceito foi elaborado em várias rodadas de ação, reflexão e discussão coletivas, com base nos dados gerados nas duas fases do projeto de pesquisa-ação. Neste ensaio, são apresentadas as palavras-chave em torno das quais o construto se articula (comum, cuidado, tecnologia, eficácia, sustentabilidade, instituição), ilustrando as práticas ligadas a ele e o porquê de elas serem centrais para uma transformação rumo a uma sociedade mais justa, igualitária e saudável. 


\section{Práticas do comum em saúde}

No contexto político e econômico atual, em que a privatização é promovida como a solução para serviços públicos 'insustentáveis', proteger a saúde e a equidade implica apoiar sistemas de saúde, universais, solidários, financiados publicamente e de qualidade9,10. Por outro lado, em muitos países - incluindo a Itália -, o sistema de saúde é construído em torno de uma visão individualizante e medicalizante da saúde-doença, com pouca compreensão da sua determinação social. Como reflexo disso, apesar de esforços recentes em direção a abordagens integradas e centradas nas pessoas, os sistemas de saúde permanecem altamente fragmentados e isolados do ponto de vista comunicativo, particularmente na fronteira (desfocada) entre questões sociais e de saúde. Ainda centrados em torno dos hospitais e do tratamento de doenças, com poucas ou nenhuma abordagem comunitária proativa e promoção de programas de saúde, os sistemas tradicionais de saúde tendem a negligenciar as necessidades de quem não tem acesso a eles (por motivos econômicos, jurídicos, geográficos, culturais ou outros), e de quem formalmente tem acesso, mas não vê as próprias necessidades levadas em consideração ou atendidas (minorias, incluindo a comunidade LGBTQI, por exemplo). Eles também não têm os recursos, em termos de competência e de organização, para atender às complexas necessidades produzidas pelas condições de crescente precariedade social, política e econômica.

Essa tensão entre a necessidade de defender o Sistema Nacional de Saúde Italiano (SSN) do desmantelamento e da privatização e a luta por uma abordagem diversa à saúde e ao sistema de saúde, que seja mais inclusiva, acessível, justa e orientada tanto para os determinantes sociais quanto individuais da saúde, foi percebida pelo Grup-pa como um eixo central da fragmentação entre os movimentos italianos que - mais ou menos diretamente - lidam com questões de saúde. De um lado, existem organizações e redes tradicionais com um forte legado histórico com os partidos políticos e movimentos sociais que apoiaram a reforma do sistema de saúde italiano, em 1978, que se empenham contra os cortes no orçamento da saúde pública e a introdução de planos de saúde privados, e defendem o fortalecimento do financiamento público e da prestação de serviços de saúde. Do outro lado, existem novos movimentos sociais que constroem espaços inovadores e auto-organizados para lidar com as necessidades de saúde emergentes, como, por exemplo, clínicas populares (para quem não pode ter acesso ao sistema de saúde nacional, incluindo imigrantes sem documentos, mas também aqueles que são excluídos progressivamente por causa de longas listas de espera, encerramento de serviços etc.) e consultorias queer, desenvolvidas por transfeministas e comunidades LGBTQI para atender às necessidades que não são detectadas nem atendidas no SSN. Esses movimentos denunciam a mercantilização da saúde e a privatização dos atendimentos de saúde por limitarem a possibilidade de agir para transformar os serviços públicos a partir de dentro, e argumentam a necessidade de estabelecer práticas radicalmente alternativas em espaços não institucionais.

As questões abordadas pelo Grup-pa através da pesquisa-ação eram centradas no desafio de imaginar um sistema de saúde que lide com a saúde e não somente com a doença, considerando e lidando com os determinantes sociais e estruturais da saúde-doença. Isso inclui abordar as dinâmicas de poder que existem também dentro do sistema de saúde, que são responsáveis por reproduzir relações desiguais entre profissionais e entre profissionais e pacientes, aceitando como 'natural' a determinação social da saúde.

A visão denominada 'práticas do comum em saúde' envolve ligar a teoria da determinação social da saúde ao conhecimento e às práticas gerados no campo do comum, e refere-se não à posse coletiva de algo, mas à ação coletiva 
de compartilhar como um princípio político de transformação" ${ }^{11} \mathrm{O}$ foco nas práticas não é meramente estratégico (para a produção de sinergias e alianças), mas inerentemente político (considerar a participação como um valor) e conectado à saúde e à manutenção da saúde (de indivíduos e da comunidade).

O construto 'práticas do comum em saúde' pretende tornar visível uma área de agência coletiva e transformação contínua tanto nos novos espaços de saúde auto-organizados criados pelos movimentos sociais como nas ações mais tradicionais de defesa dos serviços públicos já existentes, desde que abordem a saúde como uma questão sociopolítica. O desafio é ir além da prática e da retórica da defesa da saúde pública, posicionando-se, ao mesmo tempo, fortemente contra o seu desmantelamento e privatização, e experimentar novas formas, práticas e abordagens à saúde dentro e fora das instituições existentes.

\section{Repensando o cuidado}

O conceito de 'cuidado' engloba significados diferentes e possivelmente conflitantes. Dentro do paradigma biomédico dominante, o 'cuidado' é visto frequentemente com uma ação unidirecional que um profissional dirige a um paciente, que é receptor passivo (sujeito) da ação terapêutica. Essa abordagem também é criticada dentro da medicina convencional, e hoje muitos tentam passar de uma abordagem que tem a doença no seu centro (voltada à cura) para uma abordagem que leva em conta não somente as dimensões biológicas, mas, também, as percepções do paciente e as implicações da doença no seu bem-estar psicológico e nos seus sentimentos (voltada ao cuidado). Entretanto, essa mudança de 'cura' para ‘cuidado' é somente um dos desafios/tensões envolvidos ao adotar diferentes modelos de cuidado. A ideia de um cuidado centrado nas pessoas, onde o paciente não é apenas uma pessoa com sintomas ou com uma doença, implica abordar, também, a assimetria na relação de cuidado, que é particularmente relevante para a saúde e pode levar à objetificação, infantilização e à violência estrutural.

Nessa perspectiva, é importante reconhecer como alguns movimentos sociais lutam contra a ideia de que o cuidado depende inteiramente do conhecimento técnico de um esperto/ profissional, enquanto o conhecimento e a experiência das pessoas ou da comunidade é descreditado. Entretanto, superar a hierarquia entre conhecimento técnico e científico, visto como verdadeiro e oficial/institucional, e qualquer outro tipo de conhecimento experiencial é particularmente difícil, e o paradigma dominante continua a operar também sobre/ dentro dos movimentos que lutam para se emancipar dele.

Para lidar com essa assimetria na prática, alguns grupos, especialmente de comunidades transfeministas e LGBTQI, adotam uma abordagem baseada em 'comunidades/coletivos de cuidado', onde a ação de cuidado mútuo é compartilhada dentro de um grupo ligado por visões e práticas políticas. Nessa perspectiva, o cuidado torna-se um espaço intermédio, algo que beneficia as pessoas dentro do grupo e, ao mesmo tempo, representa uma ação política ou discurso mirado a transformar o conceito e as práticas de cuidado. Em outras palavras, ele atua como um meio de subjetivação (política). É importante acrescentar que, para que espaços assim se tornem um meio de libertação coletiva, eles têm que ser baseados na abertura e na autorreflexão de cada pessoa sobre si e sobre os próprios privilégios.

\section{Qual tecnologia para qual paradigma de saúde?}

Na dominante visão medicalizada e capitalista da saúde, a melhora nas condições de saúde é representada como uma consequência da construção de mais hospitais, do desenvolvimento de mais tecnologias, mais pesquisas sobre remédios, implementação de abordagens personalizadas ao atendimento médico ${ }^{\mathbf{1 2}}$. 
Entretanto, esse paradigma afasta ainda mais a atenção e os investimentos para longe das condições que influenciam uma grande proporção das nossas chances de uma vida saudável (os determinantes sociais da saúde), que são estritamente relacionados com a forma como uma sociedade é organizada e, portanto, às questões de equidade e justiça social13.

Além disso, o desenvolvimento tecnológico é intimamente relacionado ao modo capitalista de produção, que é responsável por um fardo insustentável de externalidades para o ambiente, e é conectado à crescente desigualdade e exclusão do acesso ao cuidado médico. Isso não acontece somente em países de baixa e média renda (por exemplo, falta de acesso ao tratamento do câncer para a maioria da população mundial), mas acontece cada vez mais também em países de renda alta (por exemplo, a restrição ao acesso a novos fármacos para o tratamento da hepatite $\mathrm{C}$, imposta pelo governo italiano por causa do seu alto custo $^{\mathbf{1 4}}$ ). Finalmente, a dependência do setor privado para trazer fármacos essenciais ao mercado gera conflitos de interesse perigosos (por exemplo, nos custos e prioridades dos investimentos em pesquisa). Mais cuidado não significa necessariamente mais saúde, mas quase sempre significa mais lucro ${ }^{15}$.

Outro conjunto de considerações sobre tecnologia e saúde se relaciona às percepções, ideias e experiências das pessoas, que são centrais para a relação terapêutica. Primeiro, porque o uso da tecnologia é intimamente relacionado à questão do conhecimento técnico ou especializado, abordada anteriormente. Segundo, porque as tecnologias de saúde são conectadas ao paradigma de saúde em uso e moldam as ideias e experiências em torno do cuidado e da doença. Se a doença é algo confinado ao corpo biológico, só uma tecnologia que é capaz de explorar o corpo e seus órgãos dará respostas confiáveis; em contrapartida, a própria existência dessa tecnologia contribui para reforçar a exclusão de outras variáveis que ela não é capaz de explorar. A antropologia médica sugere não separar ideias e experiências (a mente) do corpo que as vive. Neste sentido, a dependência humana da tecnologia é um fato, independente de qualquer consideração sobre a validade da tecnologia. A tecnologia nunca pode nunca ser neutra porque ela entra nos modos segundos os quais a realidade é organizada, representada e reproduzida ${ }^{16}$.

Se, como mencionado acima, o objetivo é trabalhar para uma transformação da abordagem à saúde, tanto dentro dos serviços de saúde quanto através das novas práticas de saúde auto-organizadas dos movimentos sociais, existem três questões em aberto que devem ser respondidas:

1.É possível separar a tecnologia (médica) do modo capitalista de produção que é responsável por perpetuar a desigualdade e devastar o planeta?

2. Enquanto se critica a ideia que vê a tecnologia e a hiperespecialização como a abordagem-padrão à saúde-doença (que pode traduzir-se em um sistema de controle opressivo), mira-se excluir a tecnologia das práticas de atendimento sanitário, apesar dos comprovados benefícios de ferramentas como, por exemplo, os medicamentos essenciais?

3. Quem pode avaliar quais tecnologias são benéficas e quais não são - como e em quais espaços democráticos? É possível imaginar uma gestão coletiva das tecnologias de saúde, da pesquisa à produção? E, de novo, quais são os critérios para rotular uma tecnologia como 'benéfica'?

\section{Alargar o conceito de eficácia}

Esta última pergunta sobre o que pode ser considerado benéfico implica perguntar a nós mesmos como podemos avaliar um resultado no campo da saúde, não como uma questão 
abstrata, mas como uma ferramenta para orientar as nossas práticas de saúde coletiva. Partindo do discurso dominante em torno da eficácia, há considerações a serem feitas que podem mudar ou expandir o seu significado para abranger mais dimensões de saúde.

Dentro das instituições públicas, o foco está principalmente nos conceitos de eficiência, de relação custo-benefício e de adequação. Esses conceitos, e os indicadores relevantes, foram incorporados a uma abordagem gerencial da saúde e dos atendimentos de saúde, que é muito difundida, também, dentro dos serviços públicos de saúde.

Ao lado desses critérios, existe um largo uso do conceito de 'eficácia técnica', que é a capacidade de produzir um resultado mensurável em termos de recuperação de uma doença ou de crescimento da expectativa de vida. Essa abordagem seleciona alguns parâmetros e indicadores, aqueles relacionados a aspectos que são mensuráveis e considerados objetivos (por exemplo, a taxa de sobrevivência), reproduzindo, assim, uma visão binária da saúde e da doença que é distante das experiências vividas pelas pessoas e pode reforçar as dinâmicas de exclusão ou de controle. Além disso, considerar só os aspectos biológicos da saúde-doença pode levar à sua essencialização, ocultando as dimensões simbólicas, bem como os fatores estruturais que moldam a distribuição da saúde e da doença entre a população.

Enquanto se imaginam novas práticas de saúde, é importante imaginar, também, novas formas de olhar os resultados dos processos (de saúde) que são gerados. Nesse sentido, pode-se tentar redefinir o conceito de eficácia, para poder incluir dimensões qualitativas, subjetivas e sociopolíticas. O debate sobre como nomear tal conceito ainda está aberto. Pode-se, entretanto, usar um exemplo para ilustrar os diferentes elementos envolvidos. Do ponto de vista biomédico, a eficácia de uma intervenção em uma pessoa que sofre de tuberculose pode coincidir com a eficácia do tratamento farmacológico, apesar do fato de que este não intervém nas causas contextuais que contribuíram para o desenvolvimento da doença. Para poder definir o impacto de uma prática sanitária que atue seja na condição e na consciência individual, seja nos seus determinantes sociais, propõe-se falar de 'eficácia biopolítica', para destacar o potencial de promover a subjetivação e uma transformação geral.

\section{$O$ vértice da sustentabilidade}

A sustentabilidade representa um desafio tanto para as práticas de saúde auto-organizadas desenvolvidas pelos movimentos sociais como para aqueles que agem para mudar o sistema de saúde a partir de dentro.

Com relação ao Estado Social, o conceito ambíguo de sustentabilidade é termo perigoso. De fato, é em nome do discurso contestado de uma suposta 'insustentabilidade' que governos de todos os tipos justificam o desmantelamento de serviços públicos de saúde. Neste sentido, o conceito é usado no seu significado econômico, concentrando-se apenas nos gastos em saúde. Qualquer outra dimensão fora daquela econômica não é considerada.

Ao mesmo tempo, as novas práticas de saúde auto-organizadas dentro dos movimentos sociais têm dificuldades com a própria sustentabilidade, ainda que em bases diferentes. Eles falam de sustentabilidade referindo-se à acessibilidade e à inclusão, ao impacto, e à possibilidade de sobrevivência em um sistema capitalista, refletindo aspectos materiais e imateriais, em um nível pessoal e coletivo. Propõe-se falar da sustentabilidade das práticas do comum em saúde destacando três dimensões: material, emocional/relacional e política.

Do ponto de vista material, com quais recursos esses grupos e atividades podem contar? É possível imaginar meios de sobrevivência pessoais e coletivos que possibilitem a estabilidade e continuidade? Muitos grupos ativistas alcançados através da pesquisa-ação criam formas de renda direta ou indireta, refletem 
sobre como construir estabilidade econômica e ao mesmo tempo colocar o ativismo no centro da vida; criam práticas de redistribuição, de economia alternativa e/ou mutualismo. Qual é o impacto que essas novas formas de sustentabilidade podem ter, e quais são os seus limites em termos de acessibilidade e equidade?

Além dessas considerações, existe uma reflexão contínua sobre o que nós chamamos 'sustentabilidade emocional/relacional' dos grupos e pessoas envolvidas nesses processos. Essa segunda dimensão do conceito de sustentabilidade está ligada às práticas iniciadas para poder gerir o poder e os processos de tomada de decisão, a divisão de tarefas e responsabilidades e o nível de envolvimento pessoal e relacional. Em muitos grupos alcançados através da pesquisa-ação, é possível observar formas de autoexploração que geram desconforto e frustração, derivadas das práticas organizacionais que foram adotadas. Para passar de modelos hierárquicos para outros modelos centrados na corresponsabilidade, é necessária uma mudança também no âmbito pessoal e uma grande atenção ao cuidado dos relacionamentos, a fim de desenvolver novas formas organizacionais que mantenham juntas as necessidades, expectativas, os desejos e a execução.

Há, ainda, outra implicação da sustentabilidade, que aqui se propõe a chamar de 'sustentabilidade política'. Muitos grupos alcançados através da pesquisa-ação tentam manter juntas a ação em microcontextos, a partir das necessidades e dos desejos dos envolvidos e/ou das comunidades circundantes, com uma ação transformadora no contexto político mais amplo. Nessa tensão, há um duplo risco: reduzir a experiência coletiva às necessidades e aos desejos daqueles que (já) fazem parte dela ou, ao contrário, criar espaços e atividades distantes e destacados daqueles que os habitam.

É possível construir experiências que, partindo do indivíduo, estejam abertas a novas e diferentes necessidades, praticando uma abordagem interseccional17-19 às diferentes formas de opressão? Quais ferramentas podem ser utilizadas para avaliar o impacto dessas experiências no território e nas comunidades locais, além do impacto que elas têm na vida daqueles que as atravessam? Com quais estratégias essas lutas podem ser multiplicadas e fortalecidas, fugindo das fronteiras geográficas e dos paradigmas de posse identitária? Como planejar e organizar concretamente redes de solidariedade e alianças entre experiências?

\section{Imaginários e processos de institucionalização}

Imaginar novas práticas de saúde significa também imaginar novas instituições para a saúde e os cuidados de saúde. Para muitos movimentos sociais, o conceito de instituição está associado à disciplina, ao controle social, à padronização dos corpos e das necessidades. Contudo, está associado, também, ao conceito de 'processo de institucionalização', o processo autônomo de produção de uma instituição ${ }^{20}$.

Os processos de geração de conhecimento coletivo, as redes de bem-estar que têm origem nas comunidades, os caminhos de autocuidado que são construídos, juntos, indicam uma forma de pensar as instituições como processos vivos, abertos e participados, nos quais as práticas são negociações dinâmicas contínuas e não algo que é dado de uma vez por todas.

Nesse sentido, pode-se falar de movimentos de instituição, considerando a instituição como o poder de criar novos imaginários em oposição à natureza estática das instituições atuais. É com relação a essa linha de pensamento que foi formulada a proposta de falar sobre as novas auto-organizadas experiências sanitárias dentro dos movimentos sociais não (apenas) como práticas de autocuidado, mas como práticas do comum em saúde. Isso permite destacar seu potencial institucionalizante, que varia de acordo com a medida em que eles atribuem valor político a novos imaginários, ao mesmo tempo que escapam das formas de captura e desapropriação neoliberal. 


\section{Conclusões}

Neste ensaio, resumiram-se algumas das reflexões originadas a partir de uma pesquisa-ação conduzida 'por dentro', uma vez que o coletivo de pesquisa Grup-pa faz parte do movimento social ampliado que foi objeto da pesquisa. O discurso em torno das seis palavras-chave - comum, cuidado, tecnologia, eficácia, sustentabilidade, instituição - é, por vezes, mais desenvolvido, outras vezes, menos, com base na profundidade da análise e do intercâmbio tanto dentro do Grup-pa, como entre o coletivo e outros grupos envolvidos na experimentação de novos espaços auto-organizados em torno da saúde e dos seus determinantes.

A nomeação das 'práticas do comum em saúde' visa a superar a fragmentação entre aqueles que defendem os sistemas de saúde pública existentes e aqueles que criam novos espaços onde mais dimensões (políticas) de saúde podem ser abordadas, e abrir um campo de discussão, experimentação e ação coletiva para uma sociedade mais justa, équa e saudável. Por definição, uma pesquisa-ação gera mais perguntas do que respostas e destina-se a acompanhar uma prática de transformação. Nesse sentido, a melhor maneira de testar a validade do construto é continuar a praticá-lo.

\section{Colaboradoras}

Bodini CF (0000-0001-7629-2394)*, Bonanno G (0000-0002-3977-6020)*, Cennamo E (00000002-1046-8892)*, Mura B (0000-0003-00486867)*, Riccio M (0000-0001-5480-4694)* e Santoro L (0000-0002-7599-1916)* contribuíram igualmente para o desenvolvimento do trabalho, que é o resultado de uma pesquisa coletiva.

\section{Referências}

1. Bodini C. Il personale (medico) è politico. Salute globale e processi tras/formativi in Italia. Bolonha: Università di Bologna; 2013.

2. Camice MS, Pigiama PS. socioanalisi narrativa dell'istituzione medica. Roma: Sensibili alle foglie; 2013.

3. Global Health. Institutional [internet]. 2009. [accessed on 2019 Apr 13]. Available on: https://www.educationglobalhealth.eu/en.
4. Hochschild A. Bury the chains: prophets and rebels in the fight to free an empire's slaves. New York: Houghton Mifflin; 2005.

5. Rootes C. Environmental movements. In: Snow DA, Soule SA, Kriesi H, editors. The Blackwell Companion to Social Movements. Oxford: Blackwell; 2004. p. 608-40.

6. Heather S, Zeldes K. "Write a Chapter and Change the World” How the Boston Women's Health Book 
Collective Transformed Women's Health Then-and Now. Am. J. Publ. Health. 2008; 98(10):1741-45.

7. Grup-pa. The contribution of civil society organizations to achieving health for all - Italy [internet]. 2015. [accessed on 2019 Apr 13]. Available on: https:// gruppaphm.noblogs.org/report-di-fase-1.

8. Grup-pa. The contribution of civil society organizations to achieving health for all - Italy, phase 2 report. 2018 [internet]. 2015. [accessed on 2019 Apr 13]. Available on: https://gruppaphm.noblogs.org/report-di-fase-1.

9. Modi N, Clarke J, McKee M. Health systems should be publicly funded and publicly provided. BMJ. 2018; 10(362):k3580.

10. Sengupta A, Bodini C, Franco S. The struggle for health. Brussels: Rosa Luxemburg Stiftung, 2018. [internet]. [accessed on 2019 Apr 13]. Available on: https:// www.rosalux.eu/publications/the-struggle-for-health.

11. Dardot P, Laval C. Common: on revolution in the 21st century. Bloomsbury: Academic; 2019.

12. Illich I. Medical nemesis: the expropriation of health. New York: Pantheon Books; 1976.

13. World Health Organization. Closing the Gap in a Generation: Health Equity Through Action on the Social Determinants of Health. Geneva;Switzerland: World Health Organization; 2008.

14. SI Redazione. Epatite C. Il diritto alla cura. SaluteInternazionale [internet]. 2016. [accessed on 2019 Apr
13]. Available on: http://www.saluteinternazionale. info/2016/07/epatite-c-il-diritto-alla-cura.

15. Godlee F. Overtreatment, over here. BMJ. 2012; 3(345):e6684.

16. Mol A. The body multiple: ontology in medical practice. Durham: Duke University Press; 2002.

17. Crenshaw K. Demarginalizing the Intersection of Race and Sex: A Black Feminist Critique of Antidiscrimination Doctrine, Feminist Theory and Antiracist Politics. University of Chicago Legal Forum. 2015. [accessed on 2019 Apr 13]; 1989(1)139:167. Available on: http://chicagounbound.uchicago.edu/uclf/ vol1989/iss1/8.

18. Hill Collins P. Black feminist thought: knowledge, consciousness, and the politics of empowerment. Boston: Unwin Hyman; 1990.

19. Hancock AM. Intersectionality as a Normative and Empirical Paradigm. Polit. Gender. 2007 [accessed on 2019 Apr 13]; 3(02):248-254. Available on: http://www.journals.cambridge.org/abstract S1743923X07000062.

20. Castoriadis C. The imaginary institution of society. Cambridge: MIT Press; 1998.

Recebido em 16/05/2019

Aprovado em 15/10/2019

Conflito de interesses: inexistente

Suporte financeiro: não houve 